\title{
Catastrofização e migrânea: uma reflexão sobre o enfrentamento da dor
}

\author{
Catastrophization and migraine: a reflection on coping with pain
}

Erlene Roberta Ribeiro dos Santos'; Daniella Araújo de Oliveira²; Marcelo Moraes Valença ${ }^{3}$

${ }^{7}$ Assistant Professor, UFPE - Vitória Academic Center - Collective Health Center ${ }^{2}$ Adjunct Professor, UFPE - Physiotherapy Department - Federal University of Pernambuco, Recife, PE, Brazil ${ }^{3}$ Full Professor, UFPE - Department of Neuropsychiatry, Federal University of Pernambuco, Recife, PE, Brazil

Santos ERR, Oliveira DA, Valença MM. Catastrofização e migrânea: uma reflexão sobre o enfrentamento da dor. Headache Medicine. 2017;8(2):48-54

\section{RESUMO}

Os fatores psicológicos assumem função importante no equilíbrio e controle de situações que geram sofrimento para os indivíduos, especificamente aquelas ligadas à dor, e são reconhecidos por contribuir para o seu agravamento. Neste contexto, a catastrofização é um conjunto de pensamentos negativos em resposta à dor. Em pacientes que sofrem com a migrânea, a catastrofização pode gerar reflexos negativos para o controle e enfrentamento da dor, potencializando as crises. Objetivo: identificar o estado da arte das publicações científicas sobre a relação entre a catastrofização e migrânea.Métodos: trata-se de uma revisão integrativa da literatura, a partir da busca de artigos nas bases de dados. Na base do LILACS foram utilizados os descritores do DeCS: "cefaleia", "transtornos de enxaqueca" e "catastrofização". Nas bases Pubmed, SCOPUS e Web of Science foram utilizados os descritores do MeSH: "headache", "headache disorders primary", "Migraine disorders" e "Catastrophization". No Cinahl foram utilizados os DeCS "Headache", "headache migraine" e "headache disorder". Como critérios de elegibilidade foram selecionados todas as categorias de artigo original, revisão de literatura, reflexão, atualização, relato de experiência, estudos com indivíduos na faixa etária de 18 a 50 anos, com diagnóstico de migrânea e com sintomas de catastrofização da dor. Resultados: em um universo de 106 artigos, foram selecionados quatro que atenderam aos critérios de inclusão. Os resultados dos estudos mostram que a catastrofização da dor associada a crises de migrânea, potencializa a percepção da gravidade da mesma, colaborando para sentimentos deincapacidade para sair da crise, assim como aumento da sensação de desamparo e desespero que dificultamo controleda dor. Conclusão: indivíduos migranosos que apresentam catastrofização da dor percebem as crises de maneira mais intensa e incapacitante, o que gera impacto negativo e exerce implicações nocivas para a sua qualidade de vida.

Palavras-chave: Catastrofização; Cefaleia; Transtornos de enxaqueca

\section{ABSTRACT}

Psychological factors play an important role in the balance and control of situations that cause individual suffering, specifically those linked to pain, and are known to contribute to its aggravation. In this context, catastrophization is a set of negative thoughts in response to pain. For patients suffering from migraine, catastrophizing can generate negative reflexes for the control and coping of pain, potentiating crises. Objective: to identify the state-of-theart of scientific publication on the relationship between catastrophization and migraine. Materials and methods: This is an integrative review of literature developed from the search of papers in various databases. In LILACS, we used Portuguese descriptors of the DeCS "cefaleia", "transtornos de enxaqueca" and "catastrofização". In PubMed, SCOPUS and Web of Science, the following MeSH descriptors were uses: "headache", "Headache disorders primary", "migraine disorders" and "Catastrophization". In the CINAHL, we used DeCS descriptors "headache", "headache migraine" and "headache disorder". All categories of original papers, literature review, reflection, update, experience report, studies with individuals in the 18-50 years age group with diagnosis of migraine and symptoms of pain catastrophization were selected as eligibility criteria. Results: In a universe of 106 papers found, four that met the inclusion criteria were selected. The results of the studies show that pain catastrophization associated with migraine attacks increases the perception of the severity of migraine, contributing to feelings of inability to overcome the crisis, as well as 
increasing feelings of helplessness and despair that hinder pain control. Conclusion: Migraine-affected individuals that evidence pain catastrophization perceive crises in a more intense and incapacitating way, which has a negative effect and has harmful implications for their quality of life.

Keywords: Catastrophization; Headache; Migraine disorder

\section{INTRODUÇÃO}

A catastrofização destaca-se como um dos fatores psicossociais mais significativos na predição da intensidade da dor e da incapacidade a ela associada, relatada por pessoas com dor crônica. Trata-se de uma resposta psicológica à dor ou a sua antecipação, apresentando, portanto, uma inter-relação com a dor, focos de dor, controle e depressão, além de gerar impactos negativos na percepção da qualidade de vida. A catastrofização está caracterizada por três dimensões: a amplificação como exagero na percepção da dor, a ruminação como recorrência dos pensamentos negativos e o desamparo que fragiliza o indivíduo que necessita de apoio. ${ }^{(1)}$

As percepções catastróficas dos sintomas físicos podem criar uma percepção equivocada de sinais corporais não patológicos como sinal de doença grave. A atenção com foco nas sensações corporais, como nas dores de cabeça ou cefaleias, conduz o indivíduo a pensamentos automáticos de ameaça constante. ${ }^{(2,3)}$

A evidência aponta que indivíduos com estadiamentos mais elevados de catastrofização tendem a apresentar maior intensidade da dor, apresentando pouca confiança e capacidade para seu controle. Isto pode estar associado ao pensamento catastrófico relacionado às dores de cabeça, gerando altos níveis de ansiedade e angústia. ${ }^{(4)}$

A dor de cabeça é o distúrbio neurológico mais frequente com alta prevalência ao longo da vida dos seres humanos. Quando acontece de forma recorrente, prejudica a qualidade de vida do indivíduo. A enxaqueca ou migrânea é a sexta maior causa de incapacidade em todo o mundo. ${ }^{(5-7)}$ É uma condição de dor debilitante associada a grande deficiência pessoal e social. ${ }^{(8)}$

Pessoas com migrânea referem sensação de expectativa e medo da chegada da crise e do seu enfrentamento. Esta reação envolve diversos processos fisiológicos, cognitivos e comportamentais que, a partir da presença ou não da catastrofização, podem sofrer influência negativa e interferir na capacidade de controle da crise..$^{(2,4)}$
Diante do exposto, torna-se necessário descrever a relação entre catastrofização e migrânea abordada na literatura científica. Desta forma, o objetivo do estudo foi identificar o estado da arte das publicações científicas sobre a relação entre catastrofização em indivíduos com migrânea e seus reflexos no enfrentamento da dor. Todavia, existe uma lacuna na literatura para análise desta relação.

\section{MATERIAL E METODOS}

Trata-se de uma revisão integrativa da literatura. ${ }^{(9)}$ Entende-se por estado da arte, nesse estudo, como uma estruturação de estudos temáticos relevantes sobre os mais diversos temas nos mais variados âmbitos do conhecimento científico. ${ }^{(10)}$

O levantamento bibliográfico foi realizado no período de junho a agosto de 2017, através de consulta de artigos científicos a partir de busca nas seguintes bases de dados eletrônicas: Literatura Latino-americana e do Caribe em Ciências da Saúde (LILACS), o Pubmed, a Base referencial da Editora Elsevier (SCOPUS), o Cummulative Index to Nursing and Allied Health Literature (Cinahl) e a Web of Science.

Na base de dados LILACS, foram utilizados os descritores do DeCS: "cefaleia", "transtornos de enxaqueca" e "catastrofização". Nas bases Pubmed, SCOPUS e Web of Science foram utilizados os descritores do $\mathrm{MeSH}$ : "headache", "headachedisordersprimary", "Migraine disorders" e "Catastrophization". No Cinahl foram utilizados os DeCs "Headache", "headache migraine" e "headache disorder". Os descritores foram combinados utilizando o operador booleano AND. Não houve restrição linguística e nem de ano de publicação.

Foram adotados os seguintes critérios de seleção dos artigos: todas as categorias de artigo (original, revisão de literatura, reflexão, atualização, relato de experiência), estudos que incluíram indivíduos na faixa etária de 18 a 50 anos, com diagnóstico de migrânea e com sintomas de catastrofização da dor.

Como critérios de exclusão: artigos sobre o tema na população de crianças, adolescentes e idosos. Inicialmente foram lidos os títulos dos artigos identificados na busca, a partir dos quais foram selecionados os que preenchiam os critérios de seleção. $\bigcirc$ recurso utilizado na pesquisa foi a expressão "termo exato", associada aos descritores específicos.

Do material obtido, 106 artigos, procedeu-se uma leitura minuciosa de cada título, em seguida, do resumo/ 
artigo, de forma a destacar aqueles que responderam ao objetivo proposto por esse estudo, a fim de organizar os dados. Para tabulação dos dados, foi elaborada uma tabela de coleta de dados contendo: título, objetivos, sexo, idade, escore da escala de pensamentos catastróficos, metodologia utilizada pelo estudo e conclusões. Os artigos selecionados que preencheram todos os critérios de inclusão tiveram seus textos lidos na íntegra. Além disso, também foi feita uma busca nas referências dos artigos selecionados, por novas bibliografias. $\bigcirc$ processo de se- leção dos artigos encontra-se descrito na Figura 1. Seguindo os critérios de inclusão, 4 estudos foram selecionados para análise, os quais são referenciados no presente texto.

A análise do material foi realizada através de leitura crítica e qualitativa que permitiu identificar convergências, possibilitando o seguinte agrupamento por eixos temáticos ou categorias de análise: catastrofização e qualidade de vida; catastrofização e crises de migrânea; catastrofização e condições incapacitantes.

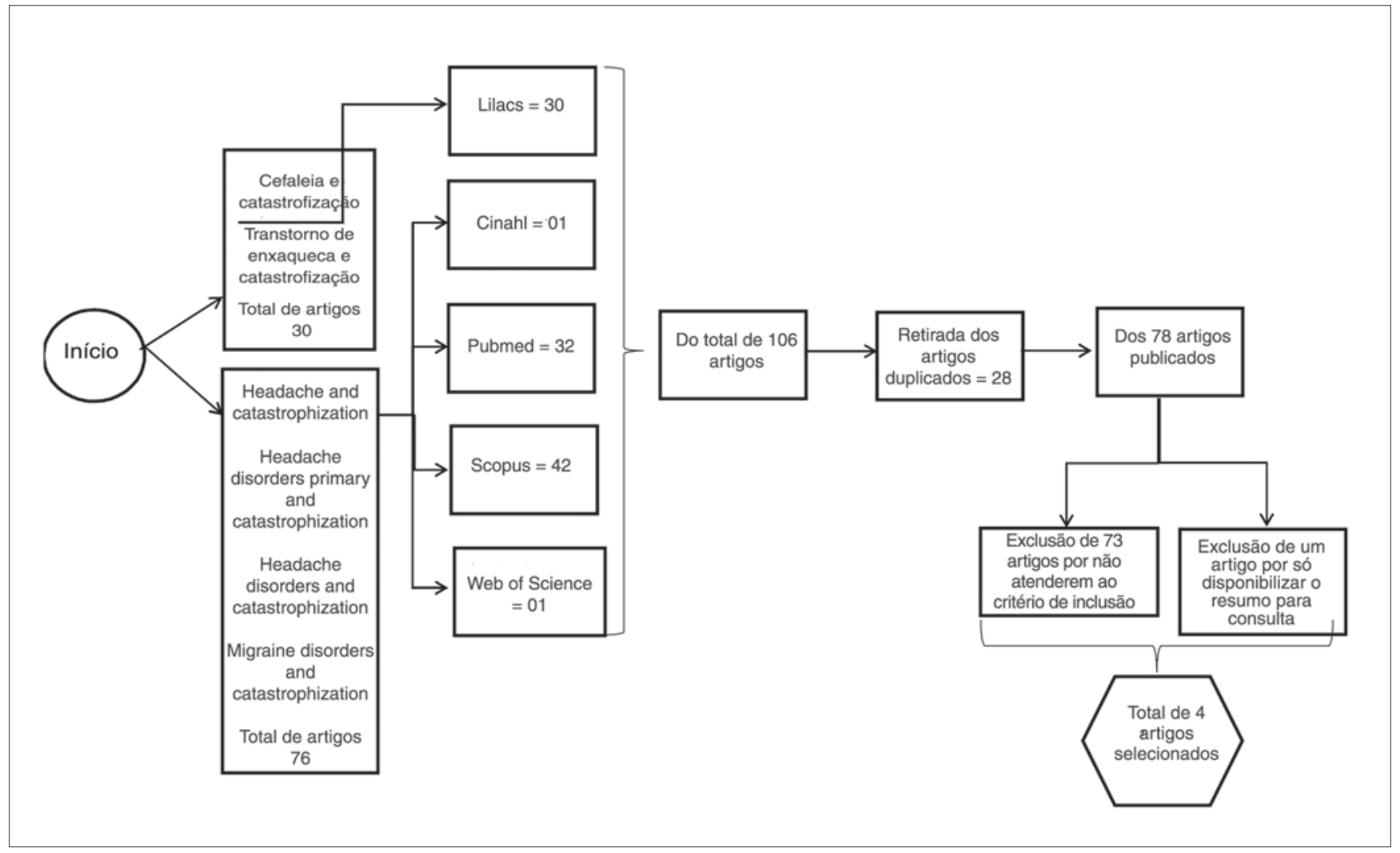

Figura 1. Processo de seleção dos artigos

\section{RESULTADOS}

Os resultados obtidos são visualizados na Tabela 1 que se segue, na qual são identificados autores, ano de publicação, objetivos, escore de pensamentos catastróficos, metodologia e conclusões.

A maioria dos participantes é constituída por mulheres, (em três dos estudos). Com relação classificação da doença, eram pacientes com diagnóstico de cefaleia (diagnosticadas por neurologista), com base na Classificação Internacional das Cefaleias, ${ }^{(7)}$ a concentração foi no tipo migrânea, associada a outras comorbidades mais frequentes, como depressão e ansiedade.

Quanto aos instrumentos de mensuração, em todos os estudos foi utilizada a Escala de Pensamentos Catastróficos PCS, associada a outras escalas, para aferir informações sobre cefaleia, impacto, incapacidade funcional, ansiedade, depressão, ideação suicida e qualidade de vida. Também foi observado, nas conclusões dos estudos, que a catastrofização da dor, associada às crises de migrânea, potencializa a percepção da gravidade, colaborando para situações mais incapacitantes para o gerenciamento das crises. 
Tabela 1. Características dos estudos selecionados que analisaram a influência da catastrofização no enfrentamento da dor em migranosos.

\begin{tabular}{|c|c|c|c|c|}
\hline Autor, ano & Objetivo & $\begin{array}{l}\text { Escore de pensamentos } \\
\text { Catastróficos }\end{array}$ & Metodologia & Conclusões \\
\hline $\begin{array}{l}\text { Holroyd } \\
\text { et al., } 2007\end{array}$ & $\begin{array}{l}\text { Examinar o } \\
\text { funcionamento e } \\
\text { a qualidade de vida } \\
\text { na migrânea grave } \\
\text { e os sintomas } \\
\text { catastróficos } \\
\text { associados à } \\
\text { ansiedade e } \\
\text { depressão. }\end{array}$ & $\begin{array}{l}\text { As variáveis catastróficas foram } \\
\text { modestas correlacionadas } \\
\text { (por exemplo, } r=0,40, P<0,001 \\
\text { para PCS e BDI). A idade e o } \\
\text { sexo foram negativamente } \\
\text { correlacionados, indicando que } \\
\text { as mulheres no estudo tendem a } \\
\text { ser mais jovens do que os } \\
\text { homens (a idade média para as } \\
\text { mulheres era de } 37 \text {, para } \\
\text { homens } 42,5 \text { ). O sexo também } \\
\text { foi associado à gravidade dos } \\
\text { sintomas associados, indicando } \\
\text { que as mulheres nesta amostra } \\
\text { relataram maior gravidade dos } \\
\text { sintomas em relação aos } \\
\text { homens. A regressão inversa } \\
\text { revelou catastrofização e } \\
\text { severidade dos sintomas } \\
\text { associados (fotofobia, } \\
\text { fonofobia, náuseas), } \\
\text { independentemente previu } \\
\text { Fn / QoL em todas as cinco } \\
\text { medidas (pesos de } 0,16 \text { a } 0,50, \\
\text { todos ( } p<0,01 \text { ). }\end{array}$ & $\begin{array}{l}232 \text { pacientes de Columbus e } \\
\text { Athems, Ohaio - USA (79\% } \\
\text { mulheres), com migrânea } \\
\text { para diagnóstico psiquiátrico } \\
\text { comórbido, completaram } \\
\text { medidas de ansiedade, } \\
\text { depressão e catastrofização, } \\
\text { registraram características } \\
\text { de migrânea em um diário e } \\
\text { preencheram cinco medidas } \\
\text { (quatro questionários de } \\
\text { autorrrelato, uma medida } \\
\text { de incapacidade diária). } \\
\text { As escalasutilizadasforam: } \\
\text { Escala de Pensamentos } \\
\text { Catastróficos (PCS); } \\
\text { Beck Anxiety Inventory (BAI); } \\
\text { Beck Depression Inventory-II } \\
\text { (BDI); Migraine-Specific } \\
\text { Quality of Life; Medical } \\
\text { Outcomes Study General } \\
\text { Health Survey; Migraine } \\
\text { Disability Assessment; } \\
\text { e Diary disability equivalent } \\
\text { hours. }\end{array}$ & $\begin{array}{l}\text { A catastrofização está } \\
\text { associada à qualidade de } \\
\text { vida, independente das } \\
\text { caracteristicas da migrânea e } \\
\text { outras variáveis demográficas } \\
\text { e psicológicas. } \\
\text { A gravidade dos sintomas } \\
\text { associados, como ansiedade } \\
\text { e depressão, também emergiu } \\
\text { como um contribuinte } \\
\text { importante para a qualidade } \\
\text { de vida dos migranosos. }\end{array}$ \\
\hline $\begin{array}{l}\text { Rathod } \\
\text { et al., } 2011\end{array}$ & $\begin{array}{l}\text { Avaliar a relação } \\
\text { entre dor, } \\
\text { incapacidade e } \\
\text { catastrofização e } \\
\text { comportamento } \\
\text { suicida em } \\
\text { pacientes com } \\
\text { cefaleia. }\end{array}$ & $\begin{array}{l}\text { Cefaleia e catastrofização } \\
\text { apresentam associação } \\
\text { significativa com ideação } \\
\text { suicida. A pontuação de } \\
\text { incapacidade foi associada } \\
\text { significativa-mente com a } \\
\text { pontuação de impedimentos } \\
(p=0,067) \text { e controlabilidade } \\
(p=0,039) \text { subescala de } \\
\text { intensidade de ideação } \\
\text { suicida. }\end{array}$ & $\begin{array}{l}200 \text { pacientes de Mysore } \\
\text { no estado de Karnataka na } \\
\text { Índia, diagnosticados com } \\
\text { cefaleia foram recrutadas } \\
\text { a partir do ambulatório do } \\
\text { Departamento de Neurologia, } \\
\text { submetidos a entrevista } \\
\text { estruturada. Cefaleia e } \\
\text { catastrofização foram } \\
\text { avaliadas com o Henry Ford } \\
\text { Hospital dor de cabeça } \\
\text { Disabilitylnventory (IDH) e } \\
\text { Escala de Pensamentos } \\
\text { Catastróficos (PCS), } \\
\text { respectivamente, para } \\
\text { avaliação e comportamento } \\
\text { de ideação suicida, Mini } \\
\text { InternationalNeuropsychiatric } \\
\text { Interview (MINI Plus) escala } \\
\text { e The Columbia-Suicídio } \\
\text { Gravidade Rating Scale } \\
\text { (C-SSRS) foram aplicados } \\
\text { também. }\end{array}$ & $\begin{array}{l}\text { A incapacidade devido à } \\
\text { cefaleia e à catastrofização } \\
\text { da dor está associada ao } \\
\text { aumento das tendências } \\
\text { suicidas, comportamentos } \\
\text { e tambèm às tentativas } \\
\text { suicidas. Cefaleia em } \\
\text { mulheres e pacientes } \\
\text { residentes em área rural, } \\
\text { indivíduos desempregados } \\
\text { de classe socioeconômica } \\
\text { baixa necessitam de atenção } \\
\text { específica para reduzir o } \\
\text { comportamento suicida de } \\
\text { alto risco. }\end{array}$ \\
\hline
\end{tabular}


Tabela 1. Características dos estudos selecionados que analisaram a influência da catastrofização no enfrentamento da dor em migranosos

\begin{tabular}{|c|c|c|c|c|}
\hline Autor, ano & Objetivo & $\begin{array}{c}\text { Escore de pensamentos } \\
\text { Catastróficos }\end{array}$ & Metodologia & Conclusões \\
\hline $\begin{array}{l}\text { Bond } \\
\text { et al., } 2015\end{array}$ & $\begin{array}{l}\text { Avaliar mulheres } \\
\text { com enxaqueca e } \\
\text { obesidade, } \\
\text { transversalmente: } \\
\text { a) prevalência de } \\
\text { catástrofe clínica; } \\
\text { b) características } \\
\text { das pessoas com e } \\
\text { sem catástrofe } \\
\text { clínica; } \\
\text { c) associações de } \\
\text { catastrofização } \\
\text { com características } \\
\text { de migrânea. }\end{array}$ & $\begin{array}{l}\text { As pontuações totais de PCS } \\
\text { mais altas foram associadas } \\
\text { independente-mente com } \\
\text { mais dias de migrânea } \\
\beta=0,331, p=0,001) \text {, } \\
\text { duração mais longa de } \\
\text { ataque }(\beta=0,390, p<0,001) \text {, } \\
\text { maiores pontuações de HIT-6 } \\
(\beta=0,425, p<0,001) \text { e } \\
\text { menores valores de HMSE } \\
(p=-0,437, p<0,001) \text {. }\end{array}$ & $\begin{array}{l}105 \text { mulheres de Providence } \\
\text { em Rhodelsland - USA, } \\
\text { que buscam tratamento } \\
\text { para emagrecer e são } \\
\text { migranosas. Registraram a } \\
\text { atividade diária de enxaqueca } \\
\text { durante } 30 \text { dias via } \\
\text { smartphone e completaram } \\
\text { a Escala de Catástrofização } \\
\text { da Dor (PCS). A mensuração } \\
\text { utilizou catástrofe clínica e } \\
\text { foi definida como o escore } \\
\text { PCS total ?30. O Teste de } \\
\text { Impacto de Cefaleia (HIT-6), } \\
\text { a Lista de Verificação de } \\
\text { Sintomas de Alodinia } \\
\text { (ASC-12), Escala de } \\
\text { Autoeficiência de } \\
\text { Gerenciamento de Cabeça } \\
\text { (HMSE) e avaliações de } \\
\text { depressão (CES-D) e } \\
\text { ansiedade (GAD-7) também } \\
\text { foram administradas. } \\
\text { Usando as pontuações de PCS e } \\
\text { o índice de massa corporal (IMC) } \\
\text { como preditores em regressão } \\
\text { linear, modelamos uma série de } \\
\text { características de dor de cabeça } \\
\text { (isto é, dias de dor de cabeça, } \\
\text { HIT-6) como resultados. }\end{array}$ & $\begin{array}{l}\text { Um quarto dos participantes } \\
\text { com migrânea e obesidade } \\
\text { apresentam catastrofização } \\
\text { da dor. Essas sofreram crises } \\
\text { mais frequentes, com maior } \\
\text { duração, maior sensibilidade } \\
\text { à dor, maior impacto na dor } \\
\text { de cabeça e menor } \\
\text { autoeficácia } \\
\text { da gestão da dor de cabeça. } \\
\text { Em todos os participantes, } \\
\text { as pontuações da escala PCS } \\
\text { estavam relacionadas a várias } \\
\text { características de migrânea, } \\
\text { além dos efeitos da } \\
\text { obesidade. }\end{array}$ \\
\hline
\end{tabular}

\begin{tabular}{|c|c|c|c|c|}
\hline $\begin{array}{l}\text { Galioto } \\
\text { et al., } 2017\end{array}$ & $\begin{array}{l}\text { Examinar se o } \\
\text { controle inibitório } \\
\text { interage com a dor } \\
\text { catastrófica para } \\
\text { prever a intensidade } \\
\text { da migrânea, } \\
\text { frequência de } \\
\text { ataque e duração } \\
\text { em mulheres com } \\
\text { enxaqueca e } \\
\text { sobrepeso / } \\
\text { obesidade, após } \\
\text { controle de idade, } \\
\text { ansiedade, depressão } \\
\text { e indice de massa } \\
\text { corporal (IMC). }\end{array}$ & $\begin{array}{l}\text { Dor Catastrófica (média, SD) } \\
\text { Pontuação Total } 22.6 \text { (11.0) } \\
\text { Rumination } 9.1(4.3) \\
\text { Ampliação } 4.3(3.1) \\
\text { Desamparo } 9.2(4.9) . \\
\text { Testes de amostra } \\
\text { independentes não } \\
\text { revelaram diferenças } \\
\text { significativas no controle } \\
\text { inibitório ou na catástrofe } \\
\text { da dor (pontuação total e } \\
\text { subescala) entre individuos } \\
\text { com e sem aura ( } p>31) .\end{array}$ & $\begin{array}{l}128 \text { mulheres de Providence, } \\
\text { Rhodelsland - USA, na faixa } \\
\text { etária de } 18 \text { a } 50 \text { anos com } \\
\text { diagnóstico de migrânea. } \\
\text { A princípio foi necessário } \\
\text { determinar o início de suas } \\
\text { enxaquecas. Em seguida, } \\
\text { os participantes tiveram seu } \\
\text { status de peso e o uso de } \\
\text { medicação preventiva basal } \\
\text { confirmados. Questionários } \\
\text { avaliaram variáveis } \\
\text { psicológicas, como } \\
\text { depressão, ansiedade, } \\
\text { incluindo a escala de } \\
\text { catástrofização } \\
\text { da dor PCS e características } \\
\text { demográficas. Foi utilizado } \\
\text { diário da cefaleia de } 28 \text { dias } \\
\text { A equipe de pesquisa } \\
\text { projetou um aplicativo } \\
\text { para uso em smartphones, } \\
\text { foi usado para gravar os } \\
\text { dados das enxaquecas } \\
\text { durante os } 28 \text { dias. }\end{array}$ & $\begin{array}{l}\text { A catastrofização da dor está } \\
\text { associada a crises de } \\
\text { migrânea mais graves e } \\
\text { incapacitantes. } \\
\text { Por outro lado, fatores que } \\
\text { moderam esse relacionamento } \\
\text { são desconhecidos. A falha no } \\
\text { controle inibitório (IC) ou a } \\
\text { capacidade de suprimir } \\
\text { respostas automáticas ou } \\
\text { inadequadas pode ser um } \\
\text { desses fatores. Pesquisas } \\
\text { demonstram relação entre a } \\
\text { catastrofização mais alta e IC } \\
\text { inferior em amostras não- } \\
\text { migranosas; pesquisa mostra } \\
\text { IC reduzido na migrânea. } \\
\text { Portanto, examinamos se o IC } \\
\text { inferior interage com o PC } \\
\text { aumentado para prever maior } \\
\text { gravidade da enxaqueca, } \\
\text { conforme medida pela } \\
\text { intensidade da dor, frequência } \\
\text { de ataque e duração. }\end{array}$ \\
\hline
\end{tabular}




\section{DISCUSSÃO}

A revisão dos estudos evidenciou prevalência da migrânea na população de mulheres o que está em sintonia com os estudos que demonstram que esta doença é mais prevalente no sexo feminino, incapacitante, muitas vezes sem diagnóstico e tratamento adequados e, ainda, com maior frequência nos anos de maior produtividade, o que traz refleflões do ponto de vista de outras áreas que fazem parte da vida do portador (econômica, social, educacional). ${ }^{(11,12)}$

A Escala de Catastrofização da Dor - PCS foi utilizada em todos os estudos selecionados e demonstrou ser o instrumento que fornece um bom índice de catastrofização a partir das subescalas correlacionadas de ruminação, amplificação e desamparo, corroborando com as sistematizações anteriores. ${ }^{(1,13)}$

\section{Catastrofização e qualidade de vida}

A gravidade dos sintomas associados, como ansiedade e depressão, também emergiu como um contribuinte importante para a piora da qualidade de vida dos migranosos. Além disso, foi observada a coexistência de outros fatores psicossociais na vida do migranoso, desencadeando expectativa e medo, que pioram o seu quadro clínico. Estas observações ratificam outras sistematizações encontradas na literatura destacando que as migrâneas, em muitas ocasiões, podem se apresentar simultaneamente com outros sintomas e condições clínicas, as chamadas comorbidades. Isto, por vezes, pode acontecer independentemente ou estar associada às mesmas. Uma das comorbidades mais prevalentes é a depressão, outra é a ansiedade, sugerindo a ocorrência de processos de adoecimento comuns. ${ }^{(3,4,14,15)}$

Além disso, o estresse emocional é relatado como um dos principais fatores que desencadeiam as crises de migrânea, inclusive correlacionado à sua duração e intensidade. Mulheres que sofrem com migrânea apresentaram níveis excessivos de estresse, ansiedade e depressão, interferindo diretamente na sua qualidade de vida e capacidade funcional para execução das atividades do cotidiano. ${ }^{(3,14,16-18)}$

\section{Catastrofização e crises de migrânea}

Na migrânea, destacada em todos os estudos, a catastrofização da dor está associada a crises mais graves e incapacitantes. Os indivíduos sofrem crises mais frequentes, com maior duração, maior sensibilidade à dor, maior impacto e menor autoeficácia da gestão da dor de cabeça. A catastrofização está associada à qualidade de vida, independente das características da migrânea e outras variáveis demográficas e psicológicas. . $^{2,3,13)}$

\section{Catastrofização e condições incapacitantes}

Em dois dos estudos aqui discutidos ficou também evidenciada a correlação entre sedentarismo, obesidade, depressão e distúrbios de sono em pacientes com migrânea. Estudo com mulheres diagnosticadas avaliou a associação do sono, depressão e ansiedade. Apesar de ser encontrada alta frequência nos distúrbios do sono, o principal fator relacionado ao impacto da migrânea foi a gravidade dos sintomas depressivos. ${ }^{(17,19,20)}$

\section{CONCLUSÃO}

A catastrofização nas crises de migrânea exerce influência significativa na frequência, duração, maior sensibilidade à dor, maior impacto e menor autoeficácia na gestão da dor de cabeça. A sensação de medo aumenta e exacerba a ansiedade na expectativa das crises, podendo desencadear outros fatores psicossociais como depressão e estresse, na maioria dos casos. Estas condições colaboram para o maior impacto negativo na qualidade de vida do migranoso, tornando ainda mais difícil o controle da dor, interferindo capacidade funcional dos indivíduos.

As características da migrânea estão associadas à deficiência de funcionamento e qualidade de vida, mas o impacto de outros fatores em pacientes com dor de cabeça ainda é inexplorado. A catastrofização neste contexto apresenta influência significativa para fomentar estudos acerca deste campo fértil para produção do conhecimento.

\section{REFERÊNCIAS}

1. Sullivan MJ, Bishop S, Pivik J. The pain catastrophizing scale: development and validation. Psychol Assess. 1995;7(4):52432.

2. Mercante JPP, Bernik MA, Zukerman-Guendler V, Zukerman E, Kuczynski E, Peres MFP. Comorbidade psiquiátrica diminui a qualidade de vida de pacientes com enxaqueca crônica. Arq Neuropsiquiatr. 2007;65(3 B):880-4.

3. Mascella V. Stress, sintomas de ansiedade e depressão na migrânea e cefaleia tensional. Campinas; 2011.

4. Breslau N, Schultz LR, Stewart WF, Lipton RB, Lucia VC, Welch KM. Headache and major depression: is the association specific to migraine? Neurology. 2000 Jan 25;54(2):308-13. 
5. Global Burden of Disease Study 2013 Collaborators. Global regional, and national incidence, prevalence, and years lived with disability for 301 acute and chronic diseases and injuries in 188 countries, 1990-2013: a systematic analysis for the Global Burd. Lancet. 2015 Aug 22;386(9995):743-800.

6. Steiner TJ, Birbeck GL, Jensen RH, Katsarava Z, Stovner LJ, Martelletti P. Headache disorders are third cause of disability worldwide. J Headache Pain. 2015; 16:58.

7. Headache Classification Committee of the International Headache Society (IHS). The International Classification of Headache Disorders, 3rd edition (beta version). Cephalal gia. 2013 Jul;33(9):629-808.

8. Lipton RB, Bigal ME. The epidemiologyof migraine. Am J Med. 2005 Mar; 118 Suppl 1:3S-10S.

9. Souza MT de, Silva MD da, Carvalho R de. Integrative review: what is it? How to do it? Einstein (Sao Paulo, Brazil) [Internet]. 2010;8(1):102-6. Available from: http://www.scielo.br/ scielo.php?script=sci arttext\&pid=S1679. $45082010000100102 \& \operatorname{lng}=\overline{e n} \& \mathrm{nrm}=$ iso\&tlng $=$ en

10. Ferreira NSDA. As pesquisas denominadas "estado da arte." Educ Soc. 2002;23(79):257-72.

11. Queiroz LP, Peres MFP, Kowacs F, Piovesan EJ, Ciciarelli MC, Souza JA, \& Zukerman E. Um estudo epidemiológico nacional da cefaléia no Brasil. Migrâneas \& Cefaleias. 2008; 11 :190-6.

12. Bond DS, Buse DC, Lipton RB, Thomas JG, Rathier L, Roth J, et al. Clinical Pain Catastrophizing in Women With Migraine and Obesity. Headache. 2015 Jul-Aug;55(7):923-33.

13. Holroyd KA, Drew JB, Cottrell CK, Romanek KM, Heh V. NIH Public Access. 2008;86(12):3279-88.

14. Oliveira DA, Cassiano L, Silva D, Katarinne J, De Brito C, Aleixo JDA, et al. $\bigcirc$ impacto da migrânea nas atividades de vida diária é mais incapacitante nas mulheres. 2008; 1 1 (4):253-5.
15. Coelho AT, Lorensini LM, Sida EY, Reimao R. Sleep quality, depression and anxiety in college students of last semesters in health area's courses. Neurobiologia. 2010;73(1):35-9.

16. Valença MM, Valença LPAA, Bordini C, Da Silva WF, Speciali JG. Cefaleia de "difícil controle. Migrâneas \& Cefaleias. 2003;6.

17. Ramos PDS, Costa JGGBD, Mancini RA, Gomez RS, Teixeira A. $L$, Barbosa IG. Association of depressive and anxiety symptoms with migraine severity. J Bras Psiquiatr. 2015;64(2):93-9.

18. Galioto R, O'Leary KC, Thomas JG, Demos K, Lipton RB, Gunstad J, et al. Lower inhibitory control interacts with greater pain catastrophizing to predict greater pain intensity in women with migraine and overweight/obesity. J Headache Pain [Internet]. 2017;18(1):41. Available from: http:// thejournalofheadacheandpain.springeropen.com/articles/ 10.1186/s10194-017-0748-8.

19. Rathod H, Ram D, Sundarmurthy H, Rathod S, John D. Headache disability, suicidality and pain catastrophization - Are they related. J Clin Diagn Res. 2016 Nov; 10(1 1):VC0 1-VC04.

20. Kwon JK, Chang IH. Pain, catastrophizing, and depression in chronic prostatitis/chronic pelvic pain syndrome. Int Neurourol J. 2013 Jun;17(2):48-58.

Correspondência

Erlene Roberta Ribeiro

Centro Acadêmico de Vitória de Santo Antão - CAV Universidade Federal de Pernambuco - UFPE

Vitória-Pernambuco, Brasil

Recebido: 15 de junho de 2017

Aceito: 30 de junho de 2017 